\title{
THE EXPECTED NUMBER OF COMPLEX ZEROS OF COMPLEX RANDOM POLYNOMIALS
}

\author{
KATRINA FERRIER, MICAH JACKSON, ANDREW LEDOAN, DHIR PATEL AND \\ HUONG TRAN
}

\begin{abstract}
By using the technique introduced in 1995 by Shepp and Vanderbei, we derive an exact formula for the expected number of complex zeros of a complex random polynomial due to Kac. The explicit evaluation of the average intensity function is obtained in closed form in the case of standard normal coefficients. In addition, we provide the limiting expressions for the intensity function and the expected number of zeros in open circular disks in the complex plane.
\end{abstract}

\section{Introduction and statement of results}

In 1943, after the brilliant work of Littlewood and Offord [13], Kac [10], [11] studied the distribution of the real zeros of a random polynomial whose coefficients are independent and identically distributed real standard normal random variables and obtained an explicit formula for the expected value of the number of its zeros in any measurable subset of the real numbers. Specifically, let $z$ be the complex variable $x+i y$, let $\left\{\eta_{j}\right\}_{j=0}^{n-1}$ be a sequence of independent and identically distributed real standard normal random variables defined on the probability space $(\Omega, \mathscr{A}, \operatorname{Pr})$, and let $P_{n}$ denote the random polynomial of degree $n-1$ given by its Taylor series at the origin

$$
P_{n}(z)=\sum_{j=0}^{n-1} \eta_{j} z^{j} .
$$

Kac showed, that for any measurable subset $\Lambda$ of the real numbers and any integer $n>1$, the expected number of real zeros in $\Lambda$ of $P_{n}$, denoted by

Received April 17, 2017; received in final form August 21, 2017.

This work is supported by National Science Foundation Grant DMS-1261308.

2010 Mathematics Subject Classification. 30C15, 30B20, 26C10, 60 B99. 
$\mathbb{E}\left[\nu_{n}(\Lambda)\right]$, is given by

$$
\mathbb{E}\left[\nu_{n}(\Lambda)\right]=\frac{4}{\pi} \int_{0}^{1}\left(\frac{1}{\left(1-x^{2}\right)^{2}}-\frac{(n+1)^{2} x^{2 n}}{\left(1-x^{2 n+2}\right)^{2}}\right)^{\frac{1}{2}} d x,
$$

where $\nu_{n}(\Lambda)$ stands for the number of real zeros in $\Lambda$ of $P_{n}$. (A short and elegant proof of this result was later provided in 1995 by Edelman and Kostlan [3], [4].) In addition, Kac obtained the asymptotic expansion

$$
\mathbb{E}\left[\nu_{n}(\Lambda)\right] \sim \frac{2}{\pi} \log n, \quad n \rightarrow \infty
$$

and the estimate

(See [2], Section 1.3, pp. 11-14.)

$$
\mathbb{E}\left[\nu_{n}(\Lambda)\right] \leq \log n+\frac{14}{\pi}, \quad n \geq 2 .
$$

Fifty-two years later, in a beautiful paper Shepp and Vanderbei [14] developed a method based on the argument principle for deriving explicit formulas for the expected number of complex zeros in any measurable subset $\Lambda$ of the complex plane, when the coefficients are independent and identically distributed real standard normal random variables. They showed, that if now $\nu_{n}(\Lambda)$ denotes the number of complex zeros in $\Lambda$ of $P_{n}$, then for any measurable region $\Lambda$ of the complex plane and any integer $n>1$,

$$
\mathbb{E}\left[\nu_{n}(\Lambda)\right]=\int_{\Lambda} h_{n}(x, y) d x d y+\int_{\Lambda \cap \mathbb{R}} g_{n}(x) d x,
$$

where the intensity function $h_{n}$ is given by

$$
h_{n}=\frac{B_{2} D_{0}^{2}-B_{0}\left(B_{1}^{2}-\left|A_{1}\right|^{2}\right)+B_{1}\left(A_{0} \bar{A}_{1}-\bar{A}_{0} A_{1}\right)}{\pi|z|^{2}\left(B_{0}^{2}-\left|A_{0}\right|^{2}\right)^{\frac{3}{2}}}
$$

and the intensity function $g_{n}$ is due to Kac and given by

$$
g_{n}=\frac{\left(B_{0} B_{2}-B_{1}^{2}\right)^{\frac{1}{2}}}{\pi|z| B_{0}},
$$

where the complex-valued functions $A_{k}$ are defined by

$$
A_{k}=\sum_{j=0}^{n-1} j^{k} z^{2 j}, \quad k=0,1,
$$

and the real-valued functions $B_{k}$ are defined on the complex plane by

$$
B_{k}=\sum_{j=0}^{n-1} j^{k}|z|^{2 j}, \quad k=0,1,2 .
$$

Ancillary to extending Kac's famous result, Shepp and Vanderebei designed an innovative robust zero-finding numerical algorithm that produces compelling computer plots of $h_{n}$ and hundreds of thousands of zeros from randomly generated polynomials. The computer plots are able to demonstrate 
the striking phenomenon that, as the degrees of these random polynomials become large, their zeros tend to lie very close to the unit circle. The zeros appear to be approximately uniformly distributed around the circle, when the real zeros are ignored.

The authors also obtained asymptotics of $h_{n}$ and $g_{n}$ confirming the classical result due to Hammersley [7], and pointed out that the same methods can be applied if the coefficients are assumed to be independent complex Gaussians. In this case, the intensity function does not have mass concentrated on the real axis (that is, $g_{n}=0$ ) and is rotationally invariant. As remarked by Shepp and Vanderebei and as follows from performing the calculations, one finds that, in this case, the intensity function is

$$
h_{n}=\frac{B_{0} B_{2}-B_{1}^{2}}{\pi|z|^{2} B_{0}^{2}},
$$

which equals the square of Kac's intensity function, but it has a different lead constant.

In the same vein, the aim of this paper is to apply these familiar and well-known methods to present anew the Shepp and Vanderbei result above for the expected number of complex zeros of a complex random polynomial whose formula permits an explicit evaluation of $h_{n}$ in closed form. We can summarize our main result as follows.

THEOREM 1. Let $\left\{\alpha_{j}\right\}_{j=0}^{n-1}$ and $\left\{\beta_{j}\right\}_{j=0}^{n-1}$ be sequences of independent and identically distributed real standard normal random variables defined on the probability space $(\Omega, \mathscr{A}, \mathrm{Pr})$. Suppose that the coefficients $\eta_{j}$ of the random polynomial $P_{n}$ are defined by $\eta_{j}=\alpha_{j}+i \beta_{j}$ for $j=0, \ldots, n-1$. Then for any measurable region $\Lambda$ of the complex plane and any integer $n>1$,

$$
\mathbb{E}\left[\nu_{n}(\Lambda)\right]=\int_{\Lambda} h_{n}(x, y) d x d y,
$$

where the intensity function $h_{n}$ is given by

$$
h_{n}=\frac{1}{\pi}\left(\frac{1}{\left(1-|z|^{2}\right)^{2}}-\frac{n^{2}|z|^{2 n-2}}{\left(1-|z|^{2 n}\right)^{2}}\right) .
$$

This theorem leads to the fact that $h_{n}$ has a well defined limit as $n \rightarrow \infty$.

Corollary. For any $z \neq \pm 1$,

$$
\lim _{n \rightarrow \infty} h_{n}=\frac{1}{\pi\left(1-|z|^{2}\right)^{2}}
$$

for $z= \pm 1$,

$$
h_{n}=\frac{n^{2}-1}{12 \pi} .
$$


As further applications, on the basis of the preceding theorem we derive limiting expressions for the expected number of zeros in open circular disks in the complex plane, starting with the disk $D(0, r)$ with radius $r$ centered at 0 .

TheOREM 2. For each disk $D(0, r)$ with radius $r<1$ centered at the origin,

$$
\lim _{n \rightarrow \infty} \mathbb{E}\left[\nu_{n}(D(0, r))\right]=\frac{r^{2}}{1-r^{2}} .
$$

In addition, for each disk $D\left(0, e^{-\frac{s}{2 n}}\right)$ with radius $e^{-\frac{s}{2 n}}$, where $s>0$, centered at the origin,

$$
\lim _{n \rightarrow \infty} \mathbb{E}\left[\frac{1}{n} \nu_{n}\left(D\left(0, e^{-\frac{s}{2 n}}\right)\right)\right]=1+\frac{1}{s}-\frac{1}{1-e^{-s}}
$$

\section{Proof of Theorem 1}

Without loss of generality, we may consider regions that are either regions that do not intersect the real axis or polar rectangles that do intersect the real axis. We commence by taking a region $\Lambda$ that does not intersect the real axis. If $z$ does not lie on the real axis, the argument principle yields

$$
\nu_{n}(\Lambda)=\frac{1}{2 \pi i} \int_{\partial \Lambda} \frac{P_{n}^{\prime}(z)}{P_{n}(z)} d z .
$$

Then taking expectations, using Fubini's theorem to interchange expectation and integral (a justification is provided in [8], Section 2.4.1, pp. 24-25, for a random analytic function, which can be tailored for a random polynomial; very recently, a sketch of the proof for the justification of the interchange of the expectation and the contour integral has been provided in [16], pp. 6-9), we obtain

$$
\mathbb{E}\left[\nu_{n}(\Lambda)\right]=\frac{1}{2 \pi i} \int_{\partial \Lambda} F(z) d z
$$

where $F$ is given by

$$
F(z)=\mathbb{E}\left[\frac{P_{n}^{\prime}(z)}{P_{n}(z)}\right]
$$

and can be evaluated using the calculus of residues.

In the process of dealing with this integral, it is necessary first to resolve the complex standard normal random variables $P_{n}$ and $P_{n}^{\prime}$ into their real and imaginary parts. We have

$$
\begin{aligned}
P_{n}(z) & =\sum_{j=0}^{n-1}\left(\alpha_{j} a_{j}-\beta_{j} b_{j}\right)+i \sum_{j=0}^{n-1}\left(\alpha_{j} b_{j}+\beta_{j} a_{j}\right), \\
P_{n}^{\prime}(z) & =\sum_{j=0}^{n-1}\left(\alpha_{j} c_{j}-\beta_{j} d_{j}\right)+i \sum_{j=0}^{n-1}\left(\alpha_{j} d_{j}+\beta_{j} c_{j}\right),
\end{aligned}
$$


where

$$
\begin{aligned}
& a_{j}=\operatorname{Re}\left(z^{j}\right), \quad b_{j}=\operatorname{Im}\left(z^{j}\right), \\
& c_{j}=\operatorname{Re}\left(j z^{j-1}\right), \quad d_{j}=\operatorname{Im}\left(j z^{j-1}\right) .
\end{aligned}
$$

The four random variables

$$
\begin{array}{ll}
\xi_{1}=\operatorname{Re}\left(P_{n}(z)\right), & \xi_{2}=\operatorname{Im}\left(P_{n}(z)\right), \\
\xi_{3}=\operatorname{Re}\left(P_{n}^{\prime}(z)\right), & \xi_{4}=\operatorname{Im}\left(P_{n}^{\prime}(z)\right),
\end{array}
$$

are correlated normal random variables. That they can be represented in terms of independent standard normal random variables is key. For we now introduce

$$
\xi \stackrel{\mathrm{D}}{=} L \zeta,
$$

where

$$
\xi=\left[\begin{array}{llll}
\xi_{1} & \xi_{2} & \xi_{3} & \xi_{4}
\end{array}\right]^{T}, \quad \zeta=\left[\begin{array}{llll}
\zeta_{1} & \zeta_{2} & \zeta_{3} & \zeta_{4}
\end{array}\right]^{T},
$$

and $L$ is a lower triangular Cholesky factor of

$$
\operatorname{Cov}(\xi)=\mathbb{E}\left[\xi \xi^{T}\right]=\mathbb{E}\left[L \zeta(L \zeta)^{T}\right]=L L^{T} ;
$$

that is, $L=\left[l_{i j}\right]$ for $i \geq j$, and $L=0$ for $i<j$. Here, the symbol $\stackrel{D}{=}$ is used to denote equality in distribution.

In order to be able to easily compute things, we designate

$$
\begin{aligned}
& a=\left[\begin{array}{llll}
a_{0} & a_{1} & \cdots & a_{n-1}
\end{array}\right]^{T}, \quad b=\left[\begin{array}{lllll}
b_{0} & b_{1} & \cdots & b_{n-1}
\end{array}\right]^{T}, \\
& c=\left[\begin{array}{llll}
c_{0} & c_{1} & \cdots & c_{n-1}
\end{array}\right]^{T}, \quad d=\left[\begin{array}{lllll}
d_{0} & d_{1} & \cdots & d_{n-1}
\end{array}\right]^{T} .
\end{aligned}
$$

With the notations

$$
A=\left[\begin{array}{llll}
a & b & c & d
\end{array}\right]^{T}, \quad B=\left[\begin{array}{llll}
-b & a & -d & c
\end{array}\right]^{T},
$$

the preceding covariance equation takes the matrix form

$$
A A^{T}+B B^{T}=L L^{T} .
$$

If use is made of this relation, it is found that

$$
\begin{array}{ll}
l_{11}=l_{22}=\frac{a^{T} a+b^{T} b}{\sqrt{a^{T} a+b^{T} b}}, & l_{21}=0, \\
l_{31}=l_{42}=\frac{c^{T} a+d^{T} b}{\sqrt{a^{T} a+b^{T} b}}, & l_{32}=-l_{41}=\frac{c^{T} b-d^{T} a}{\sqrt{a^{T} a+b^{T} b}} .
\end{array}
$$

Since $\xi$ and $L \zeta$ are equal in distribution,

$$
\left[\begin{array}{l}
\xi_{1} \\
\xi_{2} \\
\xi_{3} \\
\xi_{4}
\end{array}\right] \stackrel{\mathrm{D}}{=}\left[\begin{array}{c}
l_{11} \zeta_{1} \\
l_{21} \zeta_{1}+l_{22} \zeta_{2} \\
l_{31} \zeta_{1}+l_{32} \zeta_{2}+l_{33} \zeta_{3} \\
l_{41} \zeta_{1}+l_{42} \zeta_{2}+l_{43} \zeta_{3}+l_{44} \zeta_{4}
\end{array}\right] .
$$


From their respective components, we deduce that

$$
\frac{P_{n}^{\prime}(z)}{P_{n}(z)} \stackrel{\mathrm{D}}{=} \frac{\left(l_{31}+i l_{41}\right) \zeta_{1}+\left(l_{32}+i l_{42}\right) \zeta_{2}+\left(l_{33}+i l_{43}\right) \zeta_{3}+i l_{44} \zeta_{4}}{\left(l_{11}+i l_{21}\right) \zeta_{1}+i l_{22} \zeta_{2}} .
$$

At this juncture it is critical that the independence of $\zeta_{1}, \zeta_{2}, \zeta_{3}$, and $\zeta_{4}$ be exploited. We notice that, in consequence of this,

$$
F(z)=\frac{\alpha}{\delta} \mathbb{E}\left[\frac{\zeta_{1}}{\left(\frac{\gamma}{\delta}\right) \zeta_{1}+\zeta_{2}}\right]+\frac{\beta}{\gamma} \mathbb{E}\left[\frac{\zeta_{2}}{\zeta_{1}+\left(\frac{\delta}{\gamma}\right) \zeta_{2}}\right]
$$

where

$$
\begin{array}{ll}
\alpha=l_{31}+i l_{41}, & \beta=l_{32}+i l_{42}, \\
\gamma=l_{11}+i l_{21}, & \delta=i l_{22} .
\end{array}
$$

If now we interchange $\zeta_{1}$ and $\zeta_{2}$ and use the complex-valued function $f$ that is defined off the real axis by

$$
f(w)=\mathbb{E}\left[\frac{\zeta_{1}}{w \zeta_{1}+\zeta_{2}}\right]
$$

and $\frac{\gamma}{\delta}=-i$, we get

$$
F(z)=\frac{\alpha}{\delta} f(-i)+\frac{\beta}{\gamma} f(i)
$$

and it remains to treat $f(-i)$ and $f(i)$.

We set $z=r e^{i \phi}$ with $r=\left(x^{2}+y^{2}\right)^{\frac{1}{2}}$ and $\phi=\arctan \left(\frac{y}{x}\right)$, where $-\pi<\phi \leq \pi$. Since $\zeta_{1}$ and $\zeta_{2}$ are independent, we have

$$
f_{\zeta_{1} \zeta_{2}}(x, y)=\frac{1}{2 \pi} e^{-\frac{1}{2}\left(x^{2}+y^{2}\right)}
$$

which in its turn gives

satisfying

$$
f_{R \Phi}(r, \phi)=\frac{1}{2 \pi} r e^{-\frac{1}{2} r^{2}}
$$

It is readily seen that

$$
f_{R \Phi}(r, \phi)=f_{R}(r) f_{\Phi}(\phi) .
$$

$$
\begin{aligned}
f(w) & =\int_{-\infty}^{\infty} \int_{-\infty}^{\infty}\left(\frac{x}{w x+y}\right) f_{\zeta_{1} \zeta_{2}}(x, y) d x d y \\
& =\int_{-\pi}^{\pi} \int_{0}^{\infty}\left(\frac{1}{w+\tan \phi}\right) f_{R \Phi}(r, \phi) d r d \phi \\
& =\frac{1}{2 \pi} \int_{-\pi}^{\pi} \frac{d \phi}{w+\tan \phi} \\
& =\frac{1}{\pi} \int_{-\infty}^{\infty} \frac{d u}{(w+u)\left(u^{2}+1\right)}
\end{aligned}
$$

if we use the substitution $u=\tan \phi$. 
To evaluate $f(-i)$, we consider the contour $\Gamma_{R}$ consisting of the entire boundary of the large semicircle in the lower half of the complex plane. Then by Cauchy's theorem,

$$
\lim _{R \rightarrow \infty} \oint_{\Gamma_{R}} \frac{d z}{(z-i)^{2}(z+i)}=2 \pi i \tilde{r}
$$

where $\tilde{r}$ is the sum of the residues of the integrand within $\Gamma_{R}$. The only singularity is a simple pole at $z=-i$ and the corresponding residue is $-\frac{1}{4}$. Now, as $R \rightarrow \infty$, the contribution from the curved portion of $\Gamma_{R}$ disappears because of the behavior of the integrand, whereas the contribution of the diameter gives

$$
\int_{-\infty}^{\infty} \frac{d x}{(x-i)^{2}(x+i)}=\frac{\pi i}{2}
$$

We therefore find that

$$
f(-i)=\frac{i}{2} \text {. }
$$

If now we consider a similar argument and use the contour consisting of the entire boundary of the large semicircle in the upper half of the complex plane, then we find that

$$
f(i)=-\frac{i}{2}
$$

If we substitute these results in (3) and make use of $\gamma=-i \delta$ and (2), then

$$
F(z)=\frac{c^{T} b-d^{T} a+i\left(c^{T} a+d^{T} b\right)}{i\left(a^{T} a+b^{T} b\right)} .
$$

By writing $a_{j}, b_{j}, c_{j}$, and $d_{j}$ in terms of $z$ and $\bar{z}$, it can be easily calculated that

$$
\begin{aligned}
a^{T} a+b^{T} b & =\sum_{j=0}^{n-1}|z|^{2 j}, \\
c^{T} b-d^{T} a & =\sum_{j=0}^{n-1} \frac{i j}{2}\left(\frac{1}{z}-\frac{1}{\bar{z}}\right)|z|^{2 j}, \\
c^{T} a+d^{T} b & =\sum_{j=0}^{n-1} \frac{j}{2}\left(\frac{1}{z}+\frac{1}{\bar{z}}\right)|z|^{2 j},
\end{aligned}
$$

so that

$$
F(z)=\frac{\sum_{j=0}^{n-1} j|z|^{2 j}}{\sum_{j=0}^{n-1} z|z|^{2 j}} .
$$

We have

$$
\sum_{j=0}^{n-1}|z|^{2 j}=\frac{1-|z|^{2 n}}{1-|z|^{2}} .
$$


Differentiating both sides of this equation with respect to $|z|^{2}$ and then multiplying throughout by $|z|^{2}$, we obtain

$$
\sum_{j=0}^{n-1} j|z|^{2 j}=\frac{(n-1)|z|^{2 n+2}-n|z|^{2 n}+|z|^{2}}{\left(1-|z|^{2}\right)^{2}} .
$$

Thus there follows

$$
\begin{aligned}
F(z) & =\frac{n|z|^{2 n}\left(|z|^{2}-1\right)+|z|^{2}\left(1-|z|^{2 n}\right)}{z\left(1-|z|^{2}\right)\left(1-|z|^{2 n}\right)} \\
& =\frac{1}{z}\left(n-1+\frac{1}{1-|z|^{2}}-\frac{n}{1-|z|^{2 n}}\right),
\end{aligned}
$$

whence we shall write

$$
F(z, \bar{z})=\frac{1}{z}\left(n-1+\frac{1}{1-z \bar{z}}-\frac{n}{1-(z \bar{z})^{n}}\right) .
$$

Using the Stokes-Green formula for a simply connected region $D$ with a sufficiently smooth boundary $\partial D$ in the complex form

$$
\int_{\partial D}(u(z, \bar{z}) d z+v(z, \bar{z}) d \bar{z})=\iint_{D}\left(\frac{\partial}{\partial z} v(z, \bar{z})-\frac{\partial}{\partial \bar{z}} u(z, \bar{z})\right) d z d \bar{z}
$$

with $u(z, \bar{z})=\frac{1}{2 \pi i} F(z, \bar{z}), v(z, \bar{z})=0, d z d \bar{z}=-2 i d x d y$, and $D=\Lambda$, we find that

$$
\mathbb{E}\left[\nu_{n}(\Lambda)\right]=\iint_{\Lambda} \frac{1}{\pi} \frac{\partial}{\partial \bar{z}} F(z, \bar{z}) d x d y
$$

where

$$
\frac{\partial}{\partial \bar{z}} F(z, \bar{z})=\frac{1}{(1-z \bar{z})^{2}}-\frac{n^{2}(z \bar{z})^{n-1}}{\left(1-(z \bar{z})^{n}\right)^{2}} .
$$

The required expression for $h_{n}$ is finally obtained upon substituting $|z|^{2}=z \bar{z}$.

Next, we consider a polar rectangle that covers a portion of the real axis. When $\Lambda$ is the angular interval $(-\theta, \theta)$ crossed with a radial interval $\left(r_{0}, r_{1}\right)$ of the real axis, $h_{n}$ does not have mass accumulated on the real axis. For, in virtue of (1), as $\theta \rightarrow 0$,

$$
\mathbb{E}\left[\nu_{n}\left(\left(r_{0}, r_{1}\right)\right)\right]=\frac{1}{2 \pi i} \int_{r_{0}}^{r_{1}}\left(F\left(r^{-}\right)-F\left(r^{+}\right)\right) d r,
$$

where $\nu_{n}\left(\left(r_{0}, r_{1}\right)\right)$ represents the number of zeros in $\left(r_{0}, r_{1}\right)$,

$$
\begin{aligned}
& F\left(r^{-}\right)=\lim _{\substack{z \rightarrow r \\
\operatorname{Im}(z)^{r}<0}} \mathbb{E}\left[\frac{P_{n}^{\prime}(z)}{P_{n}(z)}\right], \\
& F\left(r^{+}\right)=\lim _{\substack{z \rightarrow r \\
\operatorname{Im}(z)>0}} \mathbb{E}\left[\frac{P_{n}^{\prime}(z)}{P_{n}(z)}\right] .
\end{aligned}
$$


Thus from (4)

$$
F\left(r^{-}\right)=F\left(r^{+}\right)=\frac{\sum_{j=0}^{n-1} j r^{2 j}}{\sum_{j=0}^{n-1} r^{2 j+1}}
$$

implying

$$
\mathbb{E}\left[\nu_{n}\left(\left(r_{0}, r_{1}\right)\right)\right]=0 .
$$

This completes the proof of the theorem in question.

\section{Proof of the corollary}

The proof of the corollary to Theorem 1 distinguishes two cases. First, we consider when $z \neq \pm 1$, and hence must evaluate

$$
\lim _{n \rightarrow \infty} h_{n}(z)=\frac{1}{\pi\left(1-|z|^{2}\right)^{2}}-\frac{1}{\pi|z|^{2}} \lim _{n \rightarrow \infty} \frac{n^{2}|z|^{2 n}}{\left(1-|z|^{2 n}\right)^{2}} .
$$

We encounter two alternatives. First, it may happen that, with $|z|<1$,

$$
\lim _{n \rightarrow \infty} n|z|^{n}=-\frac{1}{\log |z|} \lim _{n \rightarrow \infty}|z|^{n}=0
$$

and

$$
\lim _{n \rightarrow \infty}\left(1-|z|^{2 n}\right)=1
$$

Otherwise, since

$$
\lim _{n \rightarrow \infty} \frac{n|z|^{n}}{1-|z|^{2 n}}=\lim _{n \rightarrow \infty} \frac{n|z|^{-n}}{|z|^{-2 n}-1},
$$

we may also have, with $|z|>1$,

$$
\lim _{n \rightarrow \infty} n|z|^{-n}=\frac{1}{\log |z|} \lim _{n \rightarrow \infty} \frac{1}{|z|^{n}}=0
$$

and

$$
\lim _{n \rightarrow \infty}\left(|z|^{-2 n}-1\right)=-1
$$

In either of these instances,

$$
\lim _{n \rightarrow \infty} \frac{n|z|^{n}}{|z|^{2 n}-1}=0
$$

Second, we consider when $z= \pm 1$. Here, we use the alternative expression for $h_{n}$,

$$
h_{n}(z)=\frac{\sum_{j=0}^{n-1}|z|^{2 j} \sum_{j=0}^{n-1} j^{2}|z|^{2 j}-\left(\sum_{j=0}^{n-1} j|z|^{2 j}\right)^{2}}{\pi|z|^{2}\left(\sum_{j=0}^{n-1}|z|^{2 j}\right)^{2}},
$$

which is obtained upon substituting $|z|^{2}=z \bar{z}$ in (4), differentiating with respect to $\bar{z}$, and then substituting in (6). The required result thereby follows by setting $z= \pm 1$ and applying the appropriate power sum formulas. 


\section{Proof of Theorem 2}

Set $z=r e^{i \phi}$ in (1). We then clearly have

$$
\begin{aligned}
\mathbb{E}\left[\nu_{n}(D(0, r))\right] & =\frac{1}{2 \pi i} \int_{\partial D(0, r)} F(z) d z \\
& =\frac{1}{2 \pi} \int_{0}^{2 \pi} z F(z) d \phi .
\end{aligned}
$$

By the bounded convergence theorem,

$$
\lim _{n \rightarrow \infty} \mathbb{E}\left[\nu_{n}(D(0, r))\right]=\frac{1}{2 \pi} \int_{0}^{2 \pi} \lim _{n \rightarrow \infty} z F(z) d \phi .
$$

The first expression in (5) further simplifies to

$$
F(z)=\frac{1}{z}\left(\frac{|z|^{2}}{1-|z|^{2}}-\frac{n|z|^{2 n}}{1-|z|^{2 n}}\right)
$$

passing this to the limit and observing that $r=|z|<1$ gives

$$
\lim _{n \rightarrow \infty} z F(z)=\frac{|z|^{2}}{1-|z|^{2}}+\frac{1}{\log |z|^{2}} \lim _{n \rightarrow \infty}|z|^{2 n}=\frac{|z|^{2}}{1-|z|^{2}} .
$$

Hence setting $z=r e^{i \phi}$ and substituting the result in (7) yields

$$
\lim _{n \rightarrow \infty} \mathbb{E}\left[\nu_{n}(D(0, r))\right]=\frac{r^{2}}{1-r^{2}}
$$

Hitherto, let us set $z=r e^{i \phi}$ with $r=e^{-\frac{s}{2 n}}$ in (1) and compute that

$$
\mathbb{E}\left[\nu_{n}\left(D\left(0, e^{-\frac{s}{2 n}}\right)\right)\right]=\frac{1}{2 \pi} \int_{0}^{2 \pi} e^{-\frac{s}{2 n}+i \phi} F\left(e^{-\frac{s}{2 n}+i \phi}\right) d \phi .
$$

By the bounded convergence theorem,

$$
\lim _{n \rightarrow \infty} \mathbb{E}\left[\frac{1}{n} \nu_{n}\left(D\left(0, e^{-\frac{s}{2 n}}\right)\right)\right]=\frac{1}{2 \pi} \int_{0}^{2 \pi} \lim _{n \rightarrow \infty} \frac{1}{n} e^{-\frac{s}{2 n}+i \phi} F\left(e^{-\frac{s}{2 n}+i \phi}\right) d \phi .
$$

We see from the second expression in (5) that

$$
\lim _{n \rightarrow \infty} \frac{1}{n} e^{-\frac{s}{2 n}+i \phi} F\left(e^{-\frac{s}{2 n}+i \phi}\right)=1+\lim _{n \rightarrow \infty} \frac{1}{n\left(1-e^{-\frac{s}{n}}\right)}-\lim _{n \rightarrow \infty} \frac{1}{1-e^{-s}} .
$$

Using the Maclaurin series of the exponential function, we find that the first limit on the right-hand side equals $\frac{1}{s}$. Also, it is plain that the second limit on the right-hand side equals $\frac{1}{1-e^{-s}}$. Consequently

$$
\lim _{n \rightarrow \infty} \mathbb{E}\left[\frac{1}{n} \nu_{n}\left(D\left(0, e^{-\frac{s}{2 n}}\right)\right)\right]=1+\frac{1}{s}-\frac{1}{1-e^{-s}}
$$

as stated in the theorem. 


\section{Final remarks and suggested future research}

Our numerical computations exhibit the behavior of $h_{n}$ and the empirical distributions for $P_{n}$ for various values of $n$. In each of Figures 1-4, the picture on the left shows a gray-scale plot of $h_{n}$, and the picture on the right represents a plot of about 20,000 zeros obtained by generating a number of random polynomials and explicitly computing their zeros. As $n$ becomes large, the zeros tend to lie very close to the unit circle and seem to be approximately uniformly distributed around the unit circle. There is no jump present near the real axis. Indeed, according to the proof of the Theorem $1, h_{n}$ does not have mass concentrated on the real axis. In Figure 4, the picture on the left seems to also capture the behavior of the limit of $h_{n}$ as $n \rightarrow \infty$. As far as we can tell, it appears to be indistinguishable from the graph of the limiting intensity function.

Finally, we mention that, in 1997, Ibragimov and Zeitouni [9] extended the result by Shepp and Vanderbei. Their method is based on an integral representation of the expected number of zeros of a random field (see Adler's classical book [1], Theorem 5.1.1, p. 95). It enabled Farahmand [5] to investigate the intensity function corresponding to the number of complex solutions of the random equation $P_{n}(z)=K+i K$, where $K$ is a constant not necessarily zero, and $P_{n}$ possesses complex coefficients, thus generalizing the intensity function obtained by Shepp and Vanderbei to nonzero $K$. (See, also, Theorem 8.3 in [6], Section 8.2, pp. 148-152.)

Very recently, Vanderbei [15] introduced a generalization to the central assumption underlining the results in [14] and derived comparable explicit
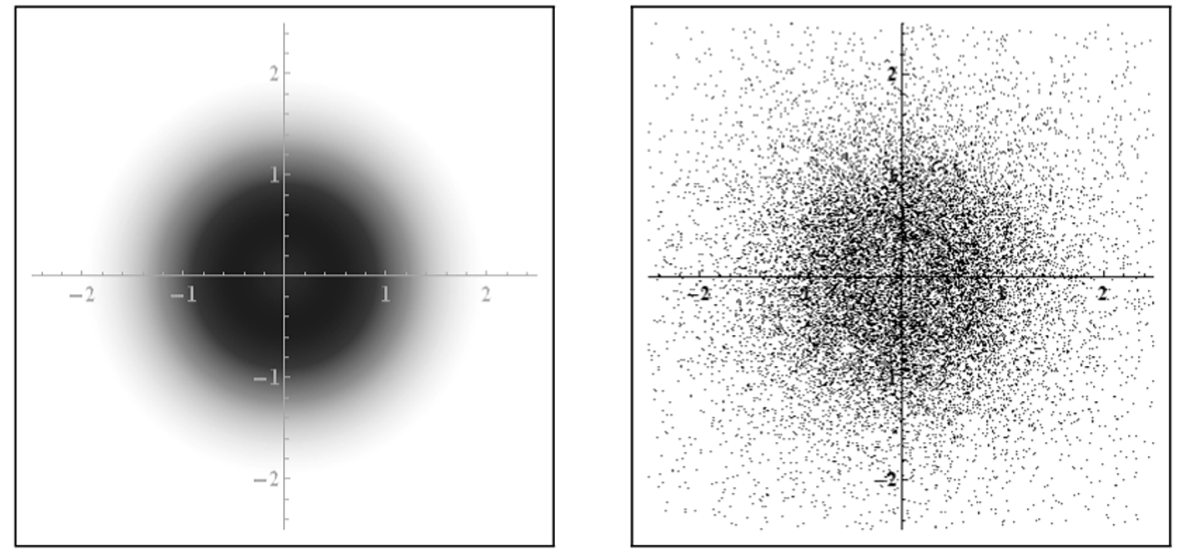

FiguRE 1. Random degree two polynomial: $\eta_{0}+\eta_{1} z+\eta_{2} z^{2}$. The empirical distribution in the right-hand plot was generated using 10,000 random polynomials. 

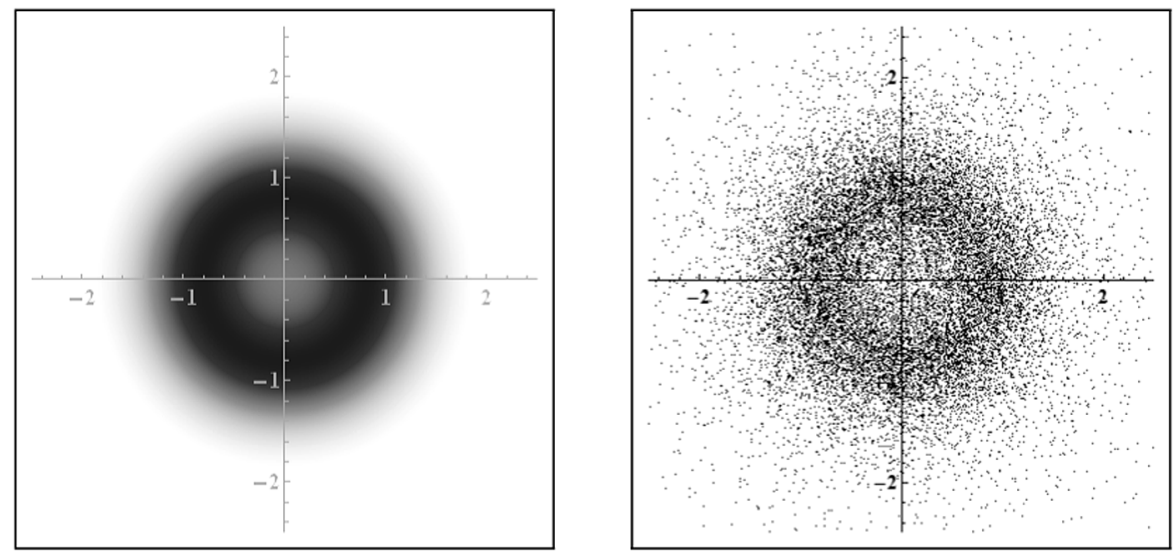

FiguRE 2. Random degree four polynomial: $\eta_{0}+\eta_{1} z+$ $\eta_{2} z^{2}+\eta_{3} z^{3}+\eta_{4} z^{4}$. The empirical distribution in the righthand plot was generated using 5,000 random polynomials.
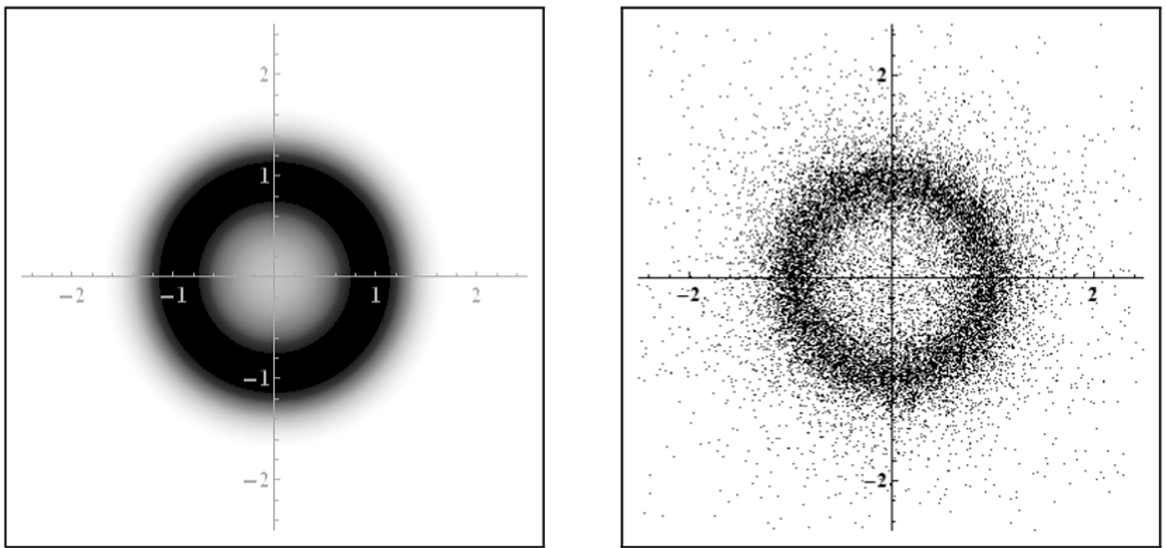

FiguRE 3. Random degree nine polynomials: $\eta_{0}+\eta_{1} z+$ $\eta_{2} z^{2}+\cdots+\eta_{9} z^{9}$. The empirical distribution in the righthand plot was generated using 2,223 random polynomials.

formulas for the distribution of zeros in the complex plane for any value of the degree of $P_{n}$. This motivated the subsequent studies by Yeager [16] and one of the authors [12]. As a matter of fact, Vanderbei's result applies to a wider class of random sums of the form $S_{n}(z)=\sum_{j=0}^{n} \eta_{j} f_{j}(z)$, where $\left\{\eta_{j}\right\}_{j=0}^{n}$ is a sequence of independent and identically distributed real standard normal random variables, and the functions $f_{j}$ are given analytic functions that are real-valued on the real number line. 

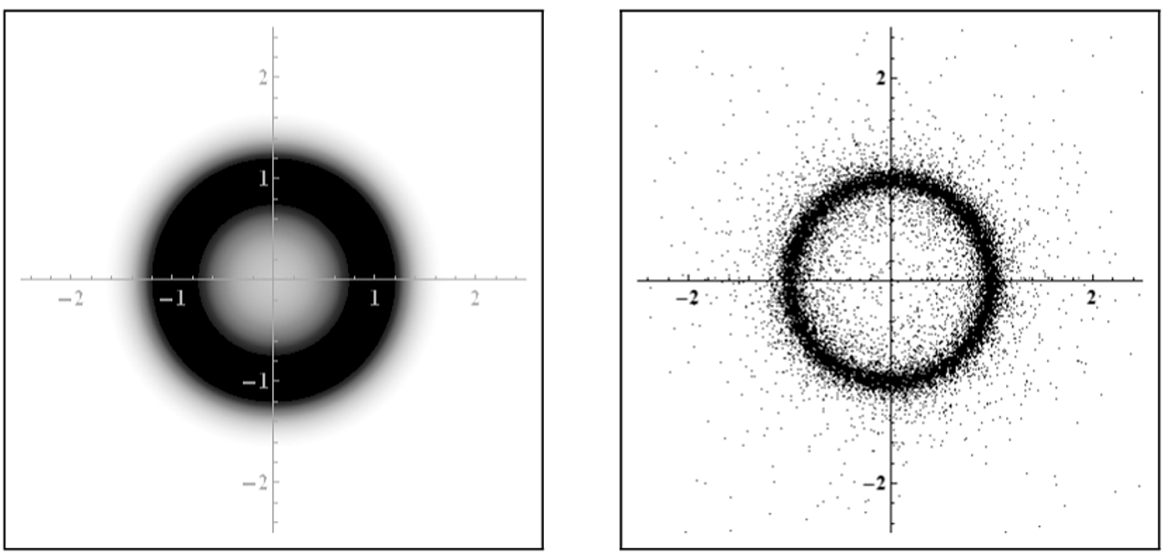

Figure 4. Random degree thirty-five polynomials: $\eta_{0}+$ $\eta_{1} z+\eta_{2} z^{2}+\cdots+\eta_{35} z^{35}$. The empirical distribution in the right-hand plot was generated using 556 random polynomials.

In connection with these works, it would be of interest to employ the technique introduced by Shepp and Vanderbei to investigate the number of $K$ level crossings of $S_{n}$. Namely, one would study the complex solutions of the random equation $S_{n}(z)=K_{1}+i K_{2}$, where $K_{1}$ and $K_{2}$ are constants not necessarily zero, and $S_{n}$ possesses either real or complex coefficients. This will be addressed in a future paper.

Acknowledgment. The authors wish to express their most sincere gratitude and appreciation to the anonymous referee for carefully reading the original version of this paper and for making a number of very helpful comments and suggestions.

\section{REFERENCES}

[1] R. J. Adler, The geometry of random fields, Wiley Ser. Probab. Math. Statist., John Wiley \& Sons, Ltd., Chichester, 1981. MR 0611857

[2] A. T. Bharucha-Reid and M. Sambandham, Random polynomials, Probab. Math. Statist., Academic Press, Inc., Orlando, Florida, 1986. MR 0856019

[3] A. Edelman and E. Kostlan, How many zeros of a random polynomial are equal?, Bull. Amer. Math. Soc. (N.S.) 32 (1995), no. 1, 1-37. MR 1290398

[4] A. Edelman and E. Kostlan, Erratum: "How many zeros of a random polynomial are real?" (Bull. Amer. Math. Soc. (N.S.) 32 (1995), no. 1, 1-37), Bull. Amer. Math. Soc. (N.S.) 33 (1996), no. 3, 325. MR 1376652

[5] K. Farahmand, Complex roots of a random algebraic polynomial, J. Math. Anal. Appl. 210 (1997), no. 2, 724-730. MR 1453202

[6] K. Farahmand, Topics in random polynomials, Pitman Res. Notes Math. Ser., vol. 393, Addison-Wesley, Longman, Edinburgh Gate, Harlow, 1998. MR 1679392 
[7] J. M. Hammersley, The zeros of a random polynomial, Proceedings of the Third Berkeley Symposium on Mathematical Statistics and Probability, 1954-1955, vol. II, University of California Press, Berkeley and Los Angeles, 1956, pp. 89-111. MR 0084888

[8] J. B. Hough, M. Krishnapur, Y. Peres and B. Virág, Zeros of Gaussian analytic functions and determinantal point processes, Univ. Lect. Ser., vol. 51, Amer. Math. Soc., Providence, Rhode Island, 2009. MR 2552864

[9] I. Ibragimov and O. Zeitouni, On roots of random polynomials, Trans. Amer. Math. Soc. 349 (1997), no. 6, 2427-2441. MR 1390040

[10] M. Kac, On the average number of real roots of a random algebraic equation, Bull. Amer. Math. Soc. 49 (1943), 314-320. MR 0007812

[11] M. Kac, A correction to "On the average number of real roots of a random algebraic equation" (Bull. Amer. Math. Soc. 49 (1943), 314-320), Bull. Amer. Math. Soc. 49 (1943), 938. MR 0009655

[12] A. Ledoan, Explicit formulas for the distribution of complex zeros of a family of random sums, J. Math. Anal. Appl. 444 (2016), no. 2, 1304-1320. MR 3535761

[13] J. E. Littlewood and A. C. Offord, On the number of real roots of a random algebraic equation, J. London Math. Soc. 13 (1938), no. 4, 288-295. MR 1574980

[14] L. A. Shepp and R. J. Vanderbei, The complex zeros of random polynomials, Trans. Amer. Math. Soc. 347 (1995), no. 11, 4365-4384. MR 1308023

[15] R. J. Vanderbei, The complex zeros of random sums, preprint, 2015; available at https://arxiv.org/pdf/1508.05162.pdf.

[16] A. M. Yeager, Zeros of random linear combinations of entire functions with complex Gaussian coefficients, preprint, 2016; available at https://arxiv.org/pdf/1605. 06836.pdf.

Katrina Ferrier, Department of Mathematics, University of Tennessee at Chattanooga, 415 EmCS Building, 615 McCallie Avenue, Chattanooga, Tennessee 37403, USA

E-mail address: klq941@mocs.utc.edu

Micah Jackson, Department of Statistics, University of California-Irvine, Bren Hall 2019, Irvine, California 92697, USA

E-mail address: gmjackso@uci.edu

Andrew Ledoan, Department of Mathematics, University of Tennessee at Chattanooga, 415 EmCS Bullding, 615 McCallie Avenue, Chattanooga, Tennessee 37403, USA

E-mail address: andrew-ledoan@utc.edu

Dhir Patel, Department of Mathematics, Ohio State University, 100 Math Tower, 231 West 18th Avenue, Columbus, Ohio 43210, USA

E-mail address: patel.2551@osu.edu

Huong Tran, Department of Mathematics, Duke University, 120 Science Drive,

Durham, North Carolina 27708, USA

E-mail address: huong.n.tran@duke.edu 